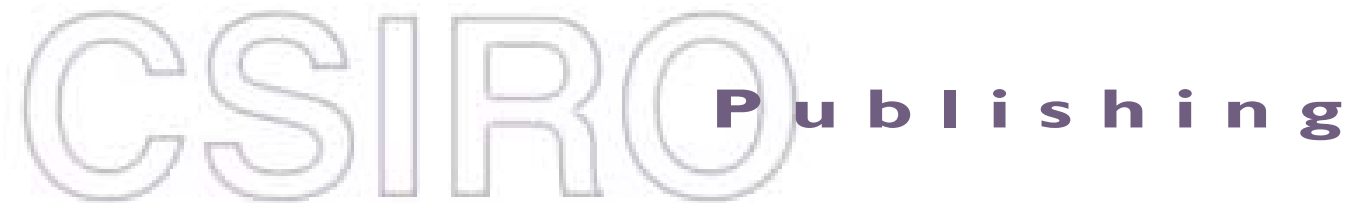

\section{Publications of the Astronomical Society of Australia}

Volume 19, 2002

(C) Astronomical Society of Australia 2002

An international journal of astronomy and astrophysics

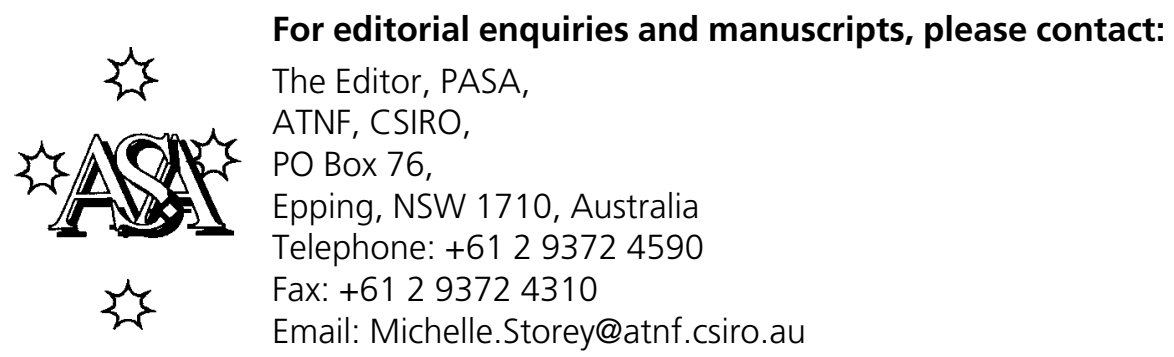

For general enquiries and subscriptions, please contact: CSIRO Publishing PO Box 1139 (150 Oxford St)

Collingwood, Vic. 3066, Australia

Telephone: +6139662 7666

Fax: +61 396627555

Email: publishing.pasa@csiro.au

C S I RO

PUBLISHING Published by CSIRO Publishing

for the Astronomical Society of Australia

www.publish.csiro.au/journals/pasa 


\title{
A Very Rapid Extreme Scattering Event in the IDV Source 0954+658
}

\author{
Giuseppe Cimò, T. Beckert, T. P. Krichbaum, L. Fuhrmann, \\ A. Kraus, A. Witzel and J. A. Zensus \\ Max-Planck-Institut für Radioastronomie, Auf dem Hügel 69, Bonn, Germany \\ gcimo@mpifr-bonn.mpg.de \\ Received 2001 August 30, accepted 2001 October 23
}

\begin{abstract}
Extreme scattering events (ESEs) are dramatic variations of the flux density at gigahertz frequencies caused by ray path distortions within an isolated inhomogeneity ('plasma lens') in the interstellar medium. These events are characterised by a deep flux density minimum in the light curve with, in some cases, surrounding maxima. The variability time scales range from weeks to months. These phenomena show a strong frequency dependence, in which the variability amplitudes increase with wavelength. During an intraday variability (IDV) monitoring project (March 2000), a feature resembling an ESE-like event appeared in the variable light curve of $0954+658$, however with a time scale of less than two days. We will discuss this effect and its implications for a better description of the interstellar medium.
\end{abstract}

Keywords: quasars: individual $(0954+658)$ — ISM: structure

\section{Introduction}

The flux densities of intraday variable radio sources (Heeschen et al. 1987) show significant variations within time scales of a few hours to $\sim 2$ days. Due to small source size, either refractive interstellar scintillation (RISS) or source intrinsic effects, or a mixture of both, are possible causes for intraday variability (IDV, see Wagner \& Witzel 1995). In addition, peculiar flux density variations with a deep minimum symmetrically surrounded by enhanced flux appearing on time scales of weeks to months were observed in some sources, and Fiedler et al. (1987) explained such variations as due to strong scattering by isolated structures in the interstellar medium. In a statistical description of this phenomenon, Fiedler et al. (1994) suggested that the identification of an ESE is difficult if the amplitude and the time scale of the variations are comparable to other possible origins of variability, and RISS may be confused with small amplitude and a very rapid ESE.

In the following we will show that we have probably detected an ESE-like event on short time scales in the multifrequency light curve of the intraday variable BL Lac object $0954+658$ with the $100 \mathrm{~m}$ radio telescope at Effelsberg in March 2000.

0954+658 was the first source in which a variability pattern resembling an ESE was seen (Fiedler et al. 1987), indicating the presence of a strongly turbulent interstellar medium along the line of sight. However, the ESE of 1981 shows variations on a time scale of $\sim 18$ weeks, much longer than the effect seen in Figure 1.

\section{Observations and Data Analysis}

In March 2000 we performed a radio-optical campaign to study possible correlations of IDV sources between different bands of the electromagnetic spectrum. The data, shown in this paper, were taken at 11,6 , and $2.8 \mathrm{~cm}$ during part of this campaign (from March 10 to 17) using the $100 \mathrm{~m}$ radio telescope of the Max-Planck-Institut für Radioastronomie (MPIfR) in Effelsberg. The data reduction was performed using CONT2, a task of the standard software package TOOLBOX of the MPIfR. Observations of non-variable sources assured a reliable flux density calibration (accuracy $\sim 0.5 \%$ ) allowing us to correct for instrumental and atmospheric effects. (Details of this data analysis can be found in Quirrenbach et al. (1992).)

In the light curve (Figure 1, top) of the BL Lac object 0954+658 $\left(m_{V}=17 \mathrm{mag}, \mathrm{z}=0.367\right.$; Stickel, Fried, \& Kuhr 1993; Padovani \& Giommi 1995), we found a systematic change of the time lag between adjacent frequencies. The first part of the light curve does not show any time lag between the three frequencies while in the last part we observe rapid and (in time) delayed changes: at JD 2451619.6, we observed a systematic time lag in the sense that the flux density at longer wavelengths peaks before the flux density at shorter wavelengths $\left(t_{11 \mathrm{~cm}}<t_{6 \mathrm{~cm}}<t_{2.8 \mathrm{~cm}}\right)$. At JD 2451620.8, the situation reversed and the shorter wavelength was peaking before the longer wavelength.

To quantify these systematic variations of the time lag, we performed a running cross correlation, i.e. time lags between 11 and $6 \mathrm{~cm}$ versus time (see Figure 1, bottom). The first part of the plot shows a flat (centred on zero) pattern indicating no time lag between 6 and $11 \mathrm{~cm}$. During the last 2 days of observations, a different behaviour is seen. We applied our model (see below) to the part of the light curve where the time lag starts to be significantly different from zero. Moreover, the cross correlation functions between 11 and $6 \mathrm{~cm}$ for the first and the last two days of observation show quite different behaviour. This again indicates that after JD 2451619.6, the variability pattern and the time lag between frequencies changed. 
Analysis of the polarisation gives further evidence for a different variability pattern before JD 2451619.6: we noted that the polarised flux density variations after JD 2451619.6 are more pronounced and faster than
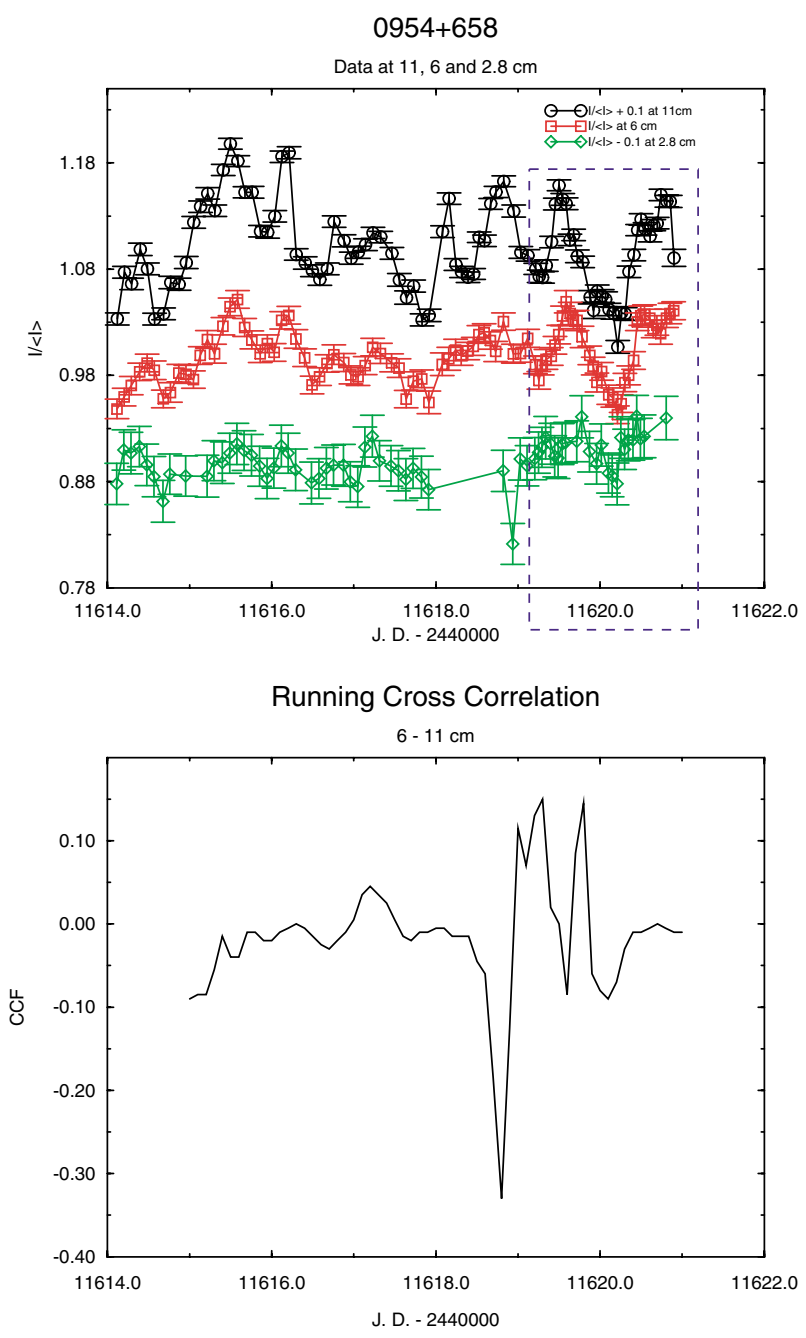

Figure 1 Top: Light curve of $0954+658$ at 11,6 , and $2.8 \mathrm{~cm}$. The dashed box highlights the ESE-like event. Bottom: Running cross correlation function (CCF), where we plot time lags versus time. We were not able to perform a proper CCF analysis for the last hours of the event because it happened at the end of our observing period. before. Using a structure function analysis, we are able to quantify this change in the polarisation characteristics of the source. The results are showed in Figure 3, where we plot structure functions and autocorrelation functions for the 2 different time intervals at $6 \mathrm{~cm}$. The typical time scale of the variations changes from $\geq 1.5$ to $\sim 0.4$ days.

\section{Description of the Model and Results}

Galactic density fluctuations along the line of sight to a compact background extragalactic source are responsible for ESEs. Such events happen usually on time scales of weeks to months. Characteristic of these events is a well defined frequency dependence: variability indices, time scales, amplitudes of variations, and amplitudes of the two surrounding maxima decrease with increasing frequency. Some of these features are similar to normal scattering processes and the shorter the time scale the more likely is the confusion of small amplitude ESEs with refractive scintillation. An important difference between an ESE and standard scattering is the focusing, which causes a reversal of the time lag: observations (Waltman et al. 1991; Fiedler et al. 1994) showed that in an ESE the variations (first maximum) start at lower frequencies. During the minimum, variations at different frequencies are simultaneous, then the variations (second maximum) start at higher frequencies (as we have also observed in our source). The two bracketing maxima are due to focusing at the edges of the cloud. A time reversal phenomenon could also appear in the weak scattering by random variations in the frequency dependence pattern of RISS (B. J. Rickett, private communication, Sydney 2001). In the case of an ESE, we suppose that such a reversal of time lags is due to changes in the optical depth of the plasma lens.

Clegg, Fey, \& Lazio (1998) developed a plasma lens model describing ESEs. We extended their model to small lenses generating very rapid ESEs. We applied such a model to the last part of our light curve (box in Figure 1), where strong variations of the time lag are seen. Figure 4 shows model light curves and cross correlations that we can immediately compare to our data. In Figure 2 we plotted the best fitting of these models versus the data at 11 and $6 \mathrm{~cm}$. At $2.8 \mathrm{~cm}$ (not shown here) the agreement is very similar.
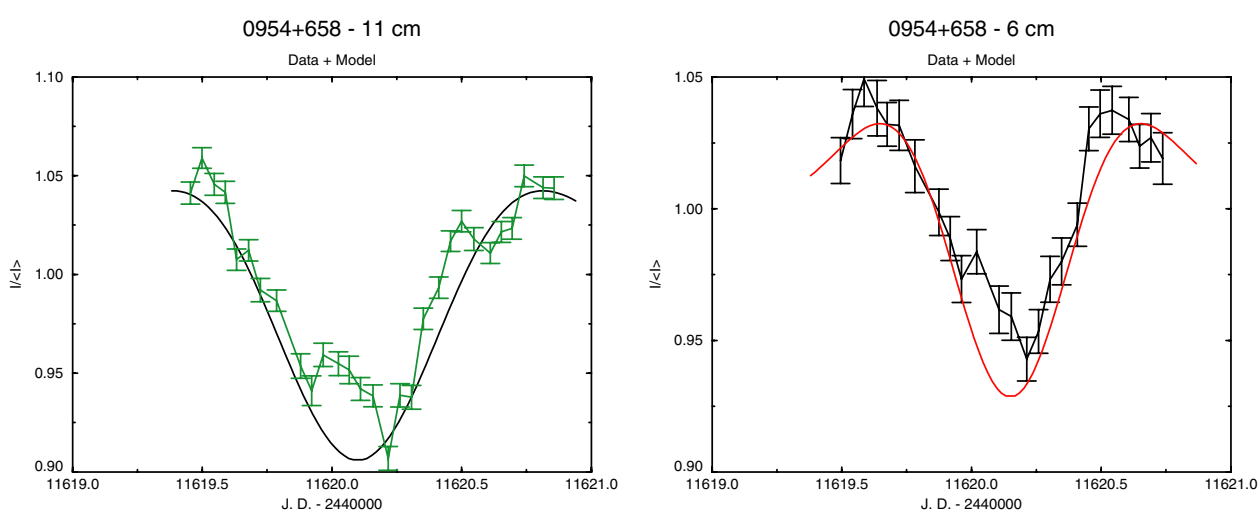

Figure 2 Normalised flux density model light curves and normalised data at 11 and $6 \mathrm{~cm}$. 

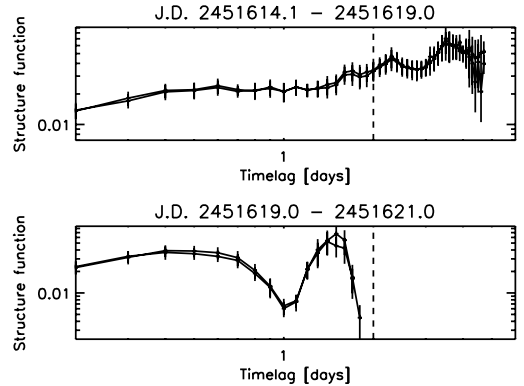

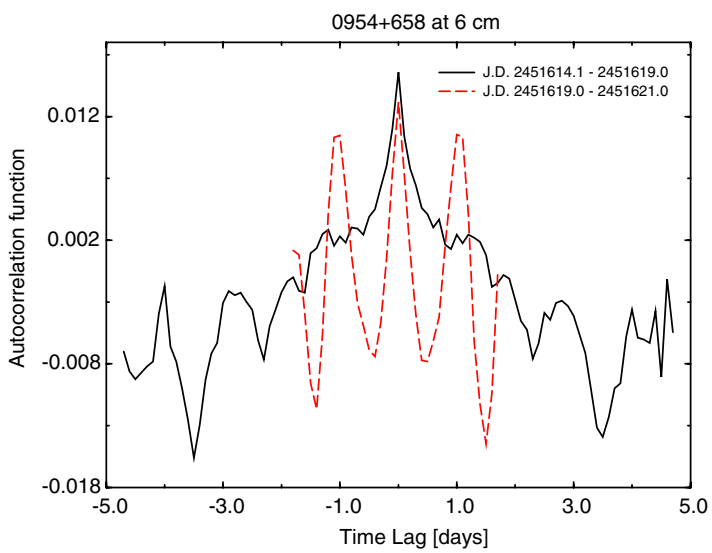

Figure 3 Structure functions and autocorrelation functions for the polarised flux density at $6 \mathrm{~cm}$ (normalised values). The minimum at 1 day (structure function at the bottom) is preserved when a longer time interval is used. This minimum directly reflects the secondary maxima in the autocorrelation function (dashed line).
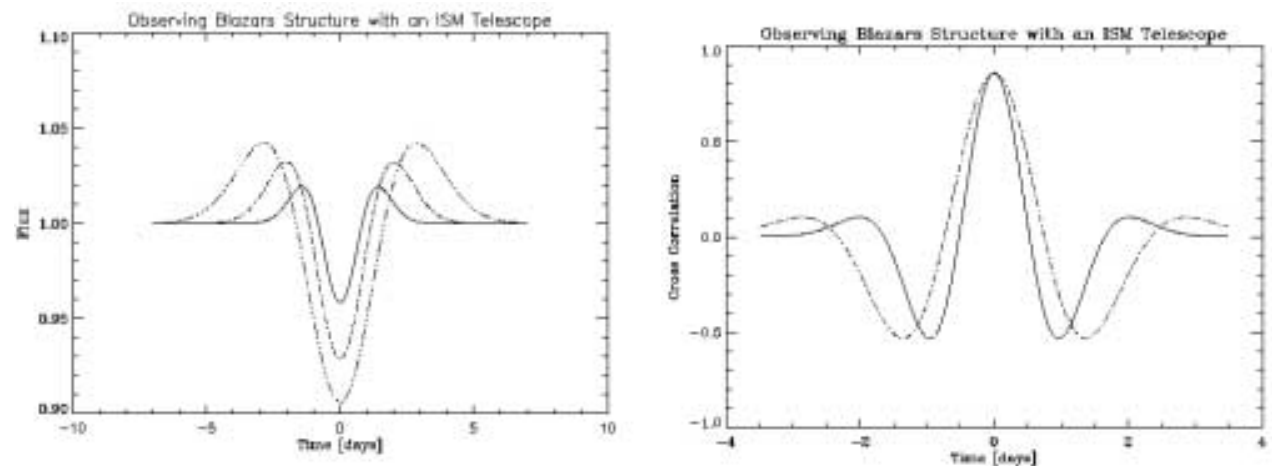

Figure 4 Left: normalised flux density model light curves of a very rapid ESE at different frequencies: $11 \mathrm{~cm}$ (dotted line), $6 \mathrm{~cm}$ (dashed-dotted line), and $3 \mathrm{~cm}$ (solid line). Right: cross correlation functions for the model light curves for a very rapid ESE between 11 and $6 \mathrm{~cm}$ (dotted line), and between 6 and $3 \mathrm{~cm}$ (solid line).

A different explanation of time reversal is given by Qian et al. (2000), who explain the time reversal in a rapid outburst of the BL Lac object $0235+164$ by relativistic aberration: a thin shock with a large Lorentz factor $(\Gamma \geq 25)$ moves along a curved magnetic field in the jet.

\section{Discussion}

Assuming a lens speed of $30 \mathrm{~km} \mathrm{~s}^{-1}$, the size of the lens would be in our case $0.035 \mathrm{AU}$. Considering the lens to be at $0.15 \mathrm{kpc}$ (distance of Galactic Loop III, direction close to the line of sight to $0954+658$ ), we can evaluate the density of the plasma lens: $N_{0} \approx 1.875 \cdot 10^{-5} \mathrm{pc} \mathrm{cm}^{-3}$ and $n_{e} \approx 110.95 \mathrm{~cm}^{-3}$, where $N_{0}$ is the column density and $n_{e}$ the electron density of the cloud.

Discussing the ESE in 1981, Clegg, Fey, \& Lazio found (at $2.25 \mathrm{GHz}$ and assuming the same distance) a lens size of $0.38 \mathrm{AU}$; lenses causing very rapid scattering events would require 10 times smaller clouds. For the standard Kolmogorov size distributions in the interstellar medium, such structures are still physically reasonable, but they put new constraints on the clumpiness of the interstellar medium.

\section{Conclusions}

Through a cross correlation analysis, we noted two effects in the IDV light curve of the source $0954+658$. We suggest that a very rapid ESE (with a time scale of 2 days) occurred in this source. The comparison of a model for ESEs and our data shows good agreement, if 10 times smaller clouds than generally accepted in the medium are assumed.

Previous studies of the IDV in this BL Lac point towards an intrinsic explanation for the rapid variations (see Wagner et al. 1993 for a radio-optical correlation evidence of its IDV), even if RISS is present due to the compactness of the source. Moreover, the polarised flux density and polarisation angle behaviour of $0954+658$ are still hard to explain by a simple RISS model. In any case, we have to take into account that a clumpy and very turbulent medium lies along the line of sight to this object. Thus a mixture of both intrinsic and extrinsic effects is a possible interpretation for such a complex behaviour.

Such a rapid ESE was never observed before. Our analysis can be repeated on previous data to discriminate between rapid ESE and other sources of variations. The above interpretation puts some constraints on the size 
(and the density) of the interstellar clouds: the time scale implies smaller clumpy structures in the interstellar medium than previously thought.

\section{References}

Clegg, A. W., Fey, A. L., \& Lazio, T. J. W. 1998, ApJ, 496, 253

Fiedler, R. L., Dennison, B., Johnston, K. J., \& Hewish, A. 1987, Nature, 326, 675

Fiedler, R. L., Dennison, B., Johnston, K. J., Waltman, E. B., \& Simon, R. S. 1994, ApJ, 430, 581
Heeschen, D. S., Krichbaum, T. P., Schalinski, C. J., \& Witzel, A. 1987, AJ, 94, 1493

Padovani, P., \& Giommi, P. 1995, MNRAS, 277, 1477

Qian, S. J., Kraus, A., Witzel, A., Krichbaum, T. P., \& Zensus, J. A. 2000, ApJ, 357, 84

Quirrenbach, A., et al. 1992, A\&A, 258, 279

Stickel, M., Fried, J. W., \& Kuhr, H. 1993, A\&AS, 98, 393

Waltman, E. B., Fiedler, R. L., Johnston, K. J., Spencer, J. H., Florkowski, D. R., Josties, F. J., McCarthy, D. D., \& Matsakis, D. N. 1991, ApJS, 77, 379

Wagner, S. J., \& Witzel, A. 1995, ARA\&A, 33, 163

Wagner, S. J., et al. 1993, A\&A, 271, 344 